\title{
Rapid intrapartum test for maternal group $B$ streptococcal colonisation and its effect on antibiotic use in labouring women with risk factors for early-onset neonatal infection (GBS2): cluster randomised trial with nested test accuracy study
}

Jane P. Daniels ${ }^{1 *}\left(\mathbb{D}\right.$, Emily Dixon², Alicia Gill², Jon Bishop², Mark Wilks ${ }^{3,4}$, Michael Millar $^{3}$, Jim Gray $^{5}$, Tracy E. Roberts ${ }^{6}$, Jane Plumb ${ }^{7}$, Jonathan J. Deeks ${ }^{6,8}$, Karla Hemming ${ }^{6}$, Khalid S. Khan ${ }^{9}$, Shakila Thangaratinam ${ }^{5,10}$ and on behalf of the GBS2 Collaborative Group

\begin{abstract}
Background: Mother-to-baby transmission of group B Streptococcus (GBS) is the main cause of early-onset infection. We evaluated whether, in women with clinical risk factors for early neonatal infection, the use of point-ofcare rapid intrapartum test to detect maternal GBS colonisation reduces maternal antibiotic exposure compared with usual care, where antibiotics are administered due to those risk factors. We assessed the accuracy of the rapid test in diagnosing maternal GBS colonisation, against the reference standard of selective enrichment culture.

Methods: We undertook a parallel-group cluster randomised trial, with nested test accuracy study and microbiological sub-study. UK maternity units were randomised to a strategy of rapid test (GeneXpert GBS system, Cepheid) or usual care. Within units assigned to rapid testing, vaginal-rectal swabs were taken from women with risk factors for vertical GBS transmission in established term labour. The trial primary outcome was the proportion of women receiving intrapartum antibiotics to prevent neonatal early-onset GBS infection. The accuracy of the rapid test was compared against the standard of selective enrichment culture in diagnosing maternal GBS colonisation. Antibiotic resistance profiles were determined in paired maternal and infant samples.
\end{abstract}

\footnotetext{
* Correspondence: jane.daniels@nottingham.ac.uk

${ }^{1}$ Nottingham Clinical Trials Unit, School of Medicine, University of Nottingham, Nottingham NG7 2RD, UK

Full list of author information is available at the end of the article
}

(c) The Author(s). 2021 Open Access This article is licensed under a Creative Commons Attribution 4.0 International License, which permits use, sharing, adaptation, distribution and reproduction in any medium or format, as long as you give appropriate credit to the original author(s) and the source, provide a link to the Creative Commons licence, and indicate if changes were made. The images or other third party material in this article are included in the article's Creative Commons licence, unless indicated otherwise in a credit line to the material. If material is not included in the article's Creative Commons licence and your intended use is not permitted by statutory regulation or exceeds the permitted use, you will need to obtain permission directly from the copyright holder. To view a copy of this licence, visit http://creativecommons.org/licenses/by/4.0/. The Creative Commons Public Domain Dedication waiver (http://creativecommons.org/publicdomain/zero/1.0/) applies to the data made available in this article, unless otherwise stated in a credit line to the data. 
Results: Twenty-two maternity units were randomised and 20 were recruited. A total of 722 mothers (749 babies) participated in rapid test units; 906 mothers (951 babies) were in usual care units. There was no evidence of a difference in the rates of intrapartum antibiotic prophylaxis (relative risk $1.16,95 \% \mathrm{Cl} 0.83$ to 1.64) between the rapid test $(41 \%, 297 / 716)$ and usual care $(36 \%, 328 / 906)$ units. No serious adverse events were reported. The sensitivity and specificity measures of the rapid test were $86 \%$ (95\% Cl 81 to 91\%) and 89\% (95\% Cl 85 to 92\%), respectively. Babies born to mothers who carried antibiotic-resistant Escherichia coli were more likely to be colonised with antibiotic-resistant strains than those born to mothers with antibiotic-susceptible E. coli.

Conclusion: The use of intrapartum rapid test to diagnose maternal GBS colonisation did not reduce the rates of antibiotics administered for preventing neonatal early-onset GBS infection than usual care, although with considerable uncertainty. The accuracy of the rapid test is within acceptable limits.

Trial registration: ISRCTN74746075. Prospectively registered on 16 April 2015

Keywords: Group B Streptococcus, Colonisation, Randomised controlled trial, Accuracy, Labour, Pregnancy, Antibiotics

\section{Background}

Group B streptococcus (GBS) is a Gram-positive pathogen found in the gut and genital tract of one in five women; a third of these women pass the bacteria to their baby during pregnancy or labour [1]. Most babies colonised with GBS are asymptomatic. GBS is one of the leading causes of early-onset neonatal infection, with a global pooled incidence rate of 0.49 per 1000 live births (95\% confidence interval [CI] 0.43 to 0.56 ) and disproportionately high mortality and morbidity $[2,3]$. In the UK and Ireland, at least two babies are diagnosed every day with GBS infection (at 0-90 days); one baby dies every week, and one survives with long-term disability [2].

In order to prevent neonatal early-onset GBS infection, pregnant women colonised with GBS are offered antibiotics intrapartum, ideally at least $4 \mathrm{~h}$ before childbirth $[4,5]$. Many countries, such as the USA, have national screening programmes that use culture-based tests to identify women colonised with GBS in late pregnancy [6]. But culture-based tests at 35-37 weeks of gestation have limited accuracy in predicting the maternal GBS colonisation status in labour $[7,8]$, take up to $48 \mathrm{~h}$ to produce a result, and are likely to be missed in women who go into labour preterm.

Some high-income countries, including the UK, remain uncertain about the balance between the benefits and harms of universal screening [9], and instead rely on a risk-based approach, where all women with risk factors are offered intrapartum antibiotic prophylaxis to prevent early-onset GBS infection in their babies [10]. Neonates are closely monitored for signs of infection and administered antibiotics if there is a diagnosis or suspicion of sepsis [11]. Since only a third of women with risk factors are colonised with GBS, a large proportion of women and their babies are unnecessarily exposed to antibiotics [12]. Rapid and accurate determination of the GBS colonisation status of the mother in labour can enable targeted administration of antibiotics in a timely fashion and reduce needless exposure to antibiotics in mothers whose babies maybe considered to be at risk of infection, but where the labouring mother is not actually colonised.

\section{Methods \\ Study aims and designs}

We undertook a cluster randomised trial to determine if the use of point-of-care intrapartum rapid test for maternal GBS colonisation, implemented at a maternity unit level, can reduce maternal and neonatal antibiotic exposure, compared with usual care where antibiotics are offered based on maternal risk factors. Testing of mothers without risk factors for early-onset infection in their babies was outside the commissioned scope of the trial.

We also assessed the real-time accuracy of a rapid nucleic acid amplification test to detect maternal GBS colonisation in women presenting to a labour ward with risk factors for neonatal early-onset GBS infection against a reference standard of selective enrichment culture in a nested test accuracy study.

In a subset of mother-child pairs, we determined the antibiotic resistance profile of any GBS isolated, and the carriage rate of other antibiotic-resistant bacteria and resistance genes in the maternal rectovaginal samples and compared the findings with the offspring's faecal sample at 6-12 weeks of age.

\section{Study setting and participants}

Twenty UK maternity units were clusters. The units were eligible to participate if they were prepared to accept a policy of rapid test-directed intrapartum antibiotic prophylaxis to prevent neonatal early-onset GBS infection and had access to microbiology facilities for 
selective enrichment bacteriological culture of GBS. Clinical midwives and doctors identified potential participants and screened for eligibility in various locations including the delivery suite, the maternity triage unit or the induction ward. No research-specific consent was obtained for the cluster trial and diagnostic study, although it was for the microbiology sub-study. Women in the rapid test units received information about the test and provided verbal assent to have the vaginal-rectal swab. Pregnant women were eligible for inclusion in the trial if they had one or more of the following risk factors: a previous baby with early- or late-onset GBS disease; GBS bacteriuria during the current pregnancy (irrespective of whether the GBS bacteriuria was treated at the time of diagnosis with antibiotics); GBS maternal colonisation of the vagina and/or rectum in the current pregnancy; suspected, diagnosed or established preterm labour (less than 37 weeks' gestation); and maternal pyrexia $\left(\geq 38^{\circ} \mathrm{C}\right)$. Women were ineligible if they were under 16 years of age, less than 24 weeks' gestation, were in the second stage of labour at admission or considered likely to give birth to their baby imminently, having a planned elective caesarean birth or their baby was known to have died in utero or had a congenital anomaly incompatible with survival at birth.

\section{Cluster randomised trial}

\section{Randomisation and masking}

Randomisation of clusters was performed at the Birmingham Clinical Trials Unit using a minimisation algorithm incorporating the following factors: region (the Midlands, London and South East England), pre-trial intrapartum antibiotic usage rate (above or below the median of all sites) and the number of vaginal or emergency Caesarean births (above or below the median). Due to the differences in the strategies for testing women and for directing intrapartum antibiotic prophylaxis, it was not possible to blind women or their care team to the randomised allocation of their maternity unit.

\section{Procedures in rapid test and usual care units}

Maternity units randomised to the rapid test received a GeneXpert $^{\circ}$ Dx IV GBS rapid testing system (Cepheid, Sunnyvale, USA) and a supply of XpertGBS test cartridges. Trained clinical midwives obtained vaginal and rectal maternal samples using a double-headed swab. One swab was used immediately for the rapid test according to the manufacturer's instructions, and a result was obtained in less than $55 \mathrm{~min}$. If the rapid test had not been initiated on the GeneXpert machine within 15 min of taking the swab, the test was considered invalid. The other swab was used for the diagnostic test accuracy study.
The rapid test units were advised to go against national guidelines, and only women who tested positive for GBS with the rapid test or for whom a test result was not available were to be offered intrapartum antibiotic prophylaxis, unless there was a clinical reason for prescribing antibiotics, or if the woman requested antibiotics.

If the woman had not given birth $48 \mathrm{~h}$ after the test result was available, the test result was regarded as invalid, and it was advised that the woman should be reswabbed and retested for GBS colonisation.

Usual care units followed their standard risk-based screening strategy where intrapartum antibiotic prophylaxis was offered to all women with risk factors.

The recommended antibiotic regimen for preventing early-onset neonatal GBS infection in both types of units in the study was in line with national recommendations, where benzyl penicillin is the first-choice antibiotic for GBS prophylaxsis [10]. Subsequent clinical management of mother and baby was based on local guidance [11].

\section{Outcome measures}

The primary outcome was the proportion of women with risk factors who received intrapartum antibiotic prophylaxis to prevent neonatal early-onset GBS infection. The secondary maternal outcomes were intrapartum maternal antibiotic administration for any indication, indications other than Caesarean section, any postpartum maternal antibiotic use and exposure to antibiotics for greater than 2 or $4 \mathrm{~h}$ before delivery. The neonatal outcomes were the proportion of newborns who receive antibiotics for any indication, with suspected or diagnosed early-onset sepsis requiring antibiotics and neonatal mortality at any time until discharge from the hospital. We also reported serious adverse events in the mother or newborn.

\section{Sample size}

The proportion of women with risk factors for earlyonset GBS infection in their newborns receiving intrapartum antibiotic prophylaxis was expected to be between 50 and 75\%, from previous estimates and expected improvements in adherence to guidelines since then [12]. With a sample size per unit of 83 women and a minimum of 20 units, we expected the trial to have $90 \%$ power to detect a reduction to $63 \%$ in rapid test units (for a comparative control of 75\%), assuming an intracluster coefficient of 0.01 [13]. This equated to a total sample size of approximately 664 per strategy group, rounded up to 1340 in total, for the cluster randomised trial. 


\section{Statistical analysis}

All trial analyses were conducted by intention-to-treat analyses according to the randomised allocation of the maternity unit, excluding any units who withdrew before data collection started and participants later found to be ineligible. For participant and cluster characteristics, we summarised the categorical data by frequencies and percentages. We summarised the continuous data by the number of responses, mean and standard deviation if deemed to be normally distributed and by the number of responses, median and interquartile range if the data appeared skewed. For the primary analysis in the cluster randomised trial, we used a mixed effects binomial regression with a log-link to estimate the relative risk, and a binomial model with an identity link to estimate the risk difference. Both models allowed for clustering by maternity unit as a random effect and adjusted for minimisation variables as fixed effects. If the binomial model with the identity link did not converge, we only reported the relative risk. In the case of non-convergence of the binomial model with a log-link, a Poisson model with robust standard errors was fitted. We used Kenward and Roger method to correct the potential inflation of the type I error rate due to the small number of clusters [14]. We used GLIMMIX in SAS to estimate model parameters, using a restricted pseudo-likelihood approach based on a marginal expansion which can be viewed as a generalised form of REML ("RMPL" option in GLIMMIX). A post hoc analysis tested sensitivity to the estimation procedure and small sample correction method by comparing results obtained from an adaptive quadrature method (with between-within small sample correction) and using a between-within correction with a restricted pseudo-likelihood marginal expansion approach (again with a between-within small sample correction) [15]. Overall inferences did not change. Where covariate adjustment was not practical, unadjusted estimates were produced and explained (e.g. not possible due to low event rate, lack of model convergence or poor recording accuracy of covariates).

We pre-specified subgroup analyses for the effects of the rapid test on the primary outcome in each of the categories based on the following maternal risk factors: maternal temperature of $38^{\circ} \mathrm{C}$ or above observed whilst in labour, previous baby with GBS disease, GBS detected in current pregnancy and preterm labour $(<37$ weeks' gestation). We summarised the treatment effects within each sub-group separately and performed an interaction test between each subgroup variable and the test strategy allocation.

\section{Diagnostic test accuracy study}

We compared the rapid test findings against the reference standard of selective enrichment culture in a cohort study nested within the randomised trial. The second vaginal-rectal swab collected for the trial was returned to the transport tube and sent to the local microbiology laboratory for selective enrichment culture to detect GBS according to the recommended methods [16]. For eligible women, a single swab taken from her baby's ear canal was also processed in the local microbiology laboratory by selective enrichment culture to detect the presence of GBS. The rapid test results preceded those of the culture test, which was interpreted blindly to the rapid test. The main test accuracy outcomes were the sensitivity and specificity of the rapid test, but we also estimated neonatal and mother-to-baby GBS transmission rates.

\section{Sample size}

For the test to be proven useful, it should detect a higher proportion of maternal GBS colonisations than other tests, but not at the cost of low specify or overdiagnosis. In the GBS1 study, intrapartum antibiotic prophylaxis directed by screening with enriched culture at 35-37 weeks' gestation was considered to be the most acceptable cost-effective strategy [12], where the sensitivity of the antenatal screening test was $75.8 \%$ (95\% CI 47.2 to 91.5\%) [17]. If the sensitivity of the proposed rapid test was higher than $90 \%$, which was the approximate upper limit of the enriched culture test sensitivity, we expected the rapid test performance to be acceptable for use in clinical practice. A sample of 676 women would have 90\% power to show that the estimated sensitivity of the rapid test was greater than a fixed value of $90 \%$, based on 167 cases of maternal GBS colonisation in the units randomised to use the rapid test and $10 \%$ failed tests.

\section{Statistical analysis}

We estimated the diagnostic accuracy of the rapid test through the standard calculations of sensitivity and specificity. Point estimates were presented with 95\% confidence intervals that were calculated using binomial exact methods [18]. We also undertook a binomial proportion test to compare the observed sensitivity with a hypothesised minimal performance value of $90 \%$.

\section{Microbiological study of bacterial antibiotic resistance}

In a subset of women from sites in London and South East England that were randomised to the rapid test strategy, we obtained individual consent to test additional vaginal-rectal maternal swabs and the faecal samples of their babies from 6 to 12 weeks of age for maternal antibiotic resistance profile of GBS, maternal colonisation by other antibiotic-resistant bacteria and the carriage of those specific bacteria or resistance elements by the infant. The swabs underwent selective enrichment culture for GBS, methicillin-resistant 
Staphylococcus aureus (MRSA), vancomycin-resistant enterococci (VRE) and extended-spectrum $\beta$-lactamase producing (ES $\beta \mathrm{L})$ Enterobacteriaceae. The presence of any bacteria of interest was profiled by a variety of techniques (including, but not necessarily limited to, antibiotic resistance, molecular/genetic characterisation and matrixassisted laser desorption ionisation time of flight mass spectrometry). Antibiotic resistance was tested using the EUCAST methods and break points [19]. Enterobacterales were tested for sensitivity to ampicillin, piperacillin and tazobactam, amoxicillin and clavulanate, cefpodoxime, gentamicin, cefuroxime, amikacin, co-trimoxazole, temocillin, ceftazidime, ertapenem and ciprofloxacin on Muller Hinton agar. Molecular testing for Gram-negative antibiotic resistance genes was performed using the GSL EasyScreen $^{\text {mix }}$ Sample Processing Kit (SP006, Genetic Signatures Ltd., Newtown, Australia) designed to rapidly isolate nucleic acids (DNA and RNA) from clinical samples via an automated purification system. Fisher's exact test was used to compare the proportions. We reported the relative risk of carriage of resistant $E$. coli or resistance genes in infants born to mothers with or without carriage of strains with specific characteristics using a binomial regression model with a log-link.

\section{Results}

Overall, we randomised 22 maternity units, of which 20 units contributed participants to the cluster randomised trial. Two sites withdrew after the randomisation and before the accrual of any data. One site that was allocated to usual care withdrew as it lost a key staff member, and the other, allocated to rapid test, then decided it could not implement the no-consent model, despite a waiver from the ethics committee. Of the 1628 eligible participants included in the dataset, 722 were in the rapid test and 906 in usual care units and gave birth between March 2018 and April 2019. There were 67 pairs of twins and 3 trios of triplets, resulting in 749 neonates in the rapid test units and 951 in the usual care units. We stopped recruitment when the sample size requirements were met. Figure 1 provides the details of the flow of participants in the trial.

\section{Characteristics of included clusters and participants}

There were ten clusters each from London, South East England and Midlands; in each region, five maternity units were randomised to the rapid test or usual care strategies. The participants were similar in age, parity and mode of delivery (Table 1).

The proportion of women with risk factors such as preterm labour and previous baby with GBS was similar. There was a higher proportion of women with a known diagnosis of GBS colonisation in current pregnancy (41\%) in the rapid test units than the usual care (31\%) units; more women had intrapartum pyrexia in the usual care units (15\%) than in the rapid test units $(8 \%)$.

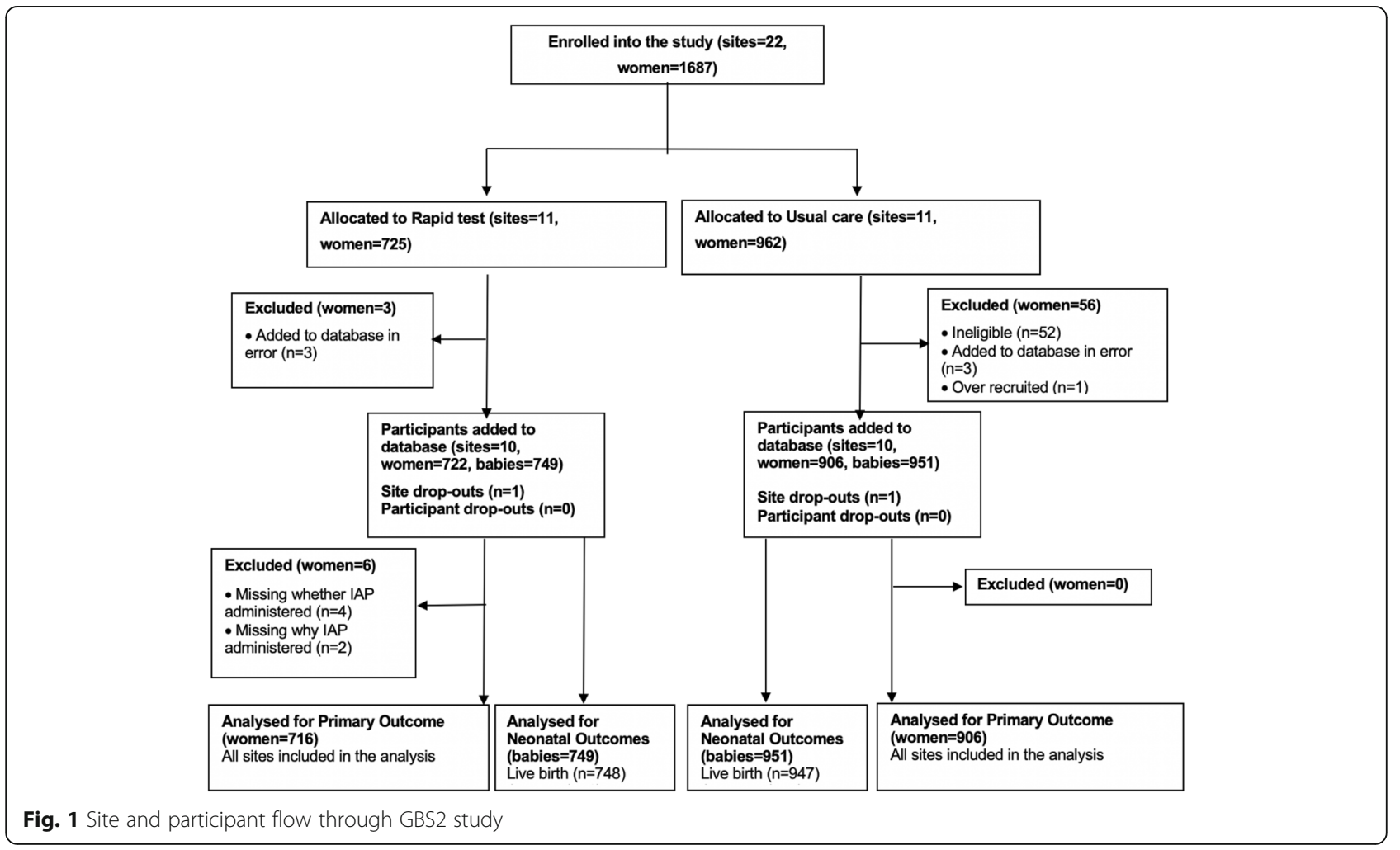


Table 1 Characteristics of clusters and participants in the GBS2 trial

\begin{tabular}{|c|c|c|c|c|}
\hline Cluster & Characteristics & $\begin{array}{l}\text { Rapid test }(n= \\
\text { 10) }\end{array}$ & $\begin{array}{l}\text { Usual care }(n= \\
10)\end{array}$ & Overall $(n=20)$ \\
\hline \multirow[t]{2}{*}{ Region } & $\begin{array}{l}\text { London and South East } \\
\text { England }\end{array}$ & $5(50 \%)$ & $5(50 \%)$ & $10(50 \%)$ \\
\hline & Midlands & $5(50 \%)$ & $5(50 \%)$ & $10(50 \%)$ \\
\hline $\begin{array}{l}\text { No. of vaginal deliveries or emergency Caesarean } \\
\text { births }\end{array}$ & Median [IQR] & 4539 [3567-5583] & 3996 [2930-5050] & 4218 [2942-5168] \\
\hline Estimated IAP rate amongst all vaginal deliveries & Median [IQR] & $25.3[22.8-32.6]$ & $27.5[9.9-31.7]$ & $26.4[13.4-32.2]$ \\
\hline Participant & Characteristics & $\begin{array}{l}\text { Rapid test }(n= \\
\text { 722) }\end{array}$ & $\begin{array}{l}\text { Usual care }(n= \\
906)\end{array}$ & $\begin{array}{l}\text { Overall }(n= \\
1628)\end{array}$ \\
\hline \multirow[t]{2}{*}{ Region } & $\begin{array}{l}\text { London and South East } \\
\text { England }\end{array}$ & $375(52 \%)$ & $458(51 \%)$ & $833(51 \%)$ \\
\hline & Midlands & $347(48 \%)$ & $448(49 \%)$ & 795 (49\%) \\
\hline \multirow[t]{2}{*}{ Age, years } & Mean (SD) & $29.3(5.8)$ & $30.1(5.8)$ & $29.7(5.8)$ \\
\hline & Missing & 1 & 0 & 1 \\
\hline \multirow[t]{3}{*}{ Onset of labour } & Spontaneous & $343(48 \%)$ & $527(58 \%)$ & $870(53 \%)$ \\
\hline & Induced & $354(49 \%)$ & $364(40 \%)$ & $718(44 \%)$ \\
\hline & Missing & $25(3 \%)$ & $15(2 \%)$ & $40(2 \%)$ \\
\hline \multirow[t]{4}{*}{ Type of delivery } & Spontaneous vaginal & $439(61 \%)$ & $542(60 \%)$ & $981(60 \%)$ \\
\hline & Instrumental & $102(14 \%)$ & $131(14 \%)$ & $233(14 \%)$ \\
\hline & Emergency Caesarean & $173(24 \%)$ & $233(26 \%)$ & $406(25 \%)$ \\
\hline & Missing & $8(1 \%)$ & $0(0 \%)$ & $8(<1 \%)$ \\
\hline \multirow[t]{3}{*}{ Multiparity } & Yes & $465(64 \%)$ & $585(65 \%)$ & $1050(65 \%)$ \\
\hline & No & $255(35 \%)$ & $321(35 \%)$ & $576(35 \%)$ \\
\hline & Missing & $2(<1 \%)$ & $0(0 \%)$ & $2(<1 \%)$ \\
\hline \multirow[t]{7}{*}{ Maternal risk factor for neonatal GBS infection } & One risk factor & $674(93 \%)$ & 841 (93\%) & $1515(93 \%)$ \\
\hline & Maternal temperature $\geq 38^{\circ} \mathrm{C}$ & $55(8 \%)$ & $139(15 \%)$ & $194(12 \%)$ \\
\hline & Previous baby with GBS & $35(5 \%)$ & $40(4 \%)$ & $75(5 \%)$ \\
\hline & GBS in this pregnancy & $293(41 \%)$ & $278(31 \%)$ & $571(35 \%)$ \\
\hline & Preterm labour & 291 (40\%) & $384(42 \%)$ & 675 (41\%) \\
\hline & Two risk factors & $46(6 \%)$ & $63(7 \%)$ & $109(7 \%)$ \\
\hline & Three risk factors & $2(<1 \%)$ & $2(<1 \%)$ & $4(<1 \%)$ \\
\hline
\end{tabular}

IAP intrapartum antibiotic prophylaxis, IQR interquartile range, $S D$ standard deviation

\section{Implementation of rapid test}

At the cluster level, we achieved complete compliance with all sites allocated to the intervention using the rapid test technology. In the 241 women who were rapid test positive, $78 \%$ (190/241) received intrapartum antibiotic prophylaxis to prevent neonatal GBS infection and $8 \%(20 / 241)$ received antibiotics for other reasons; $13 \%$ of women (31/241) who tested positive did not receive any antibiotics. Of the 316 women who were rapid test negative, $56 \%$ (176/316) received antibiotics. Of these, $17 \%$ were for prevention of neonatal GBS infection (52/316), 15\% for maternal pyrexia (48/316), 12\% prior to Caesarean section (12/316), 6\% for maternal request (19/316) and 18\% for other reasons (57/ 316). Supplementary Table S1 shows the adherence to the test result and Supplementary Table S2 the various indications for antibiotics administration.

\section{Effects of rapid intrapartum test on maternal and} neonatal exposure to antibiotics

There were no statistically significant differences in the proportion of women receiving intrapartum antibiotic prophylaxis for preventing GBS infection in the newborn (adjusted relative risk aRR 1.16, 95\% CI 0.83 to 1.64$)$ between the rapid test $(41 \%, 297 / 716)$ and the usual care $(36 \%, 328 / 906)$ units (Table 2). The overall rates of intrapartum antibiotic prophylaxis administered to the mother for any reason were also similar between the two strategies (aRR 0.99, 95\% CI 0.81 to 1.21 ). Women in the rapid test units were more likely to have received antibiotics at least $4 \mathrm{~h}$ before childbirth than those in the usual care units (adjusted risk difference aRD 0.16; 95\% CI 0.06 to 0.27) Supplementary Table S3. 
Table 2 Effects of a rapid test for GBS on maternal exposure to antibiotics compared to usual care

\begin{tabular}{|c|c|c|c|c|}
\hline Outcome & Rapid test, $n(\%)$ & Usual care, $\boldsymbol{n}(\%)$ & Adjusted risk difference $(95 \% \mathrm{Cl})^{1}$ & Adjusted relative risk $(95 \% \mathrm{Cl})^{2}$ \\
\hline Maternal primary & $(n=722)$ & $(n=906)$ & & \\
\hline \multicolumn{5}{|l|}{ IAP for GBS } \\
\hline Yes & $297(41 \%)$ & $328(36 \%)$ & $0.05(-0.07,0.18)$ & $1.16(0.83,1.64)$ \\
\hline No & $419(59 \%)$ & $578(64 \%)$ & & \\
\hline Missing & 6 & 0 & & \\
\hline \multicolumn{5}{|l|}{ Maternal secondary } \\
\hline \multicolumn{5}{|c|}{ IAP for any indication } \\
\hline Yes & $484(67 \%)$ & $602(66 \%)$ & $-0.007(-0.14,0.12)$ & $0.99(0.81,1.21)$ \\
\hline No & $238(33 \%)$ & 307 (34\%) & & \\
\hline Missing & 4 & 0 & & \\
\hline
\end{tabular}

IAP intrapartum antibiotic prophylaxis

${ }^{1}$ Risk difference; estimates $<0$ favour rapid test

${ }^{2}$ Relative risk; estimates $<1$ favour rapid test. Analyses adjusted for cluster size, unit birth rate, and estimated pre-trial IAP rates

Subgroup analysis by individual maternal risk factors did not show any statistically significant differences between the two strategies in the rates of intrapartum antibiotics administered to prevent neonatal GBS infection Supplementary Table S4. There were no reports of maternal anaphylaxis due to antibiotic administration or any reports of inoculation injury.

The neonates born to women in the rapid test units (33\%, 244/749) had a significantly lower risk of receiving antibiotics for any reason by $29 \%$ (aRR $0.71,95 \%$ CI 0.54 to 0.95$)$ than those in the usual care units $(44 \%, 412$ / 951). There was a $37 \%$ reduction in the proportion of neonates given antibiotics for suspected early-onset sepsis (aRR $0.63,95 \%$ CI 0.43 to 0.92 ) in the rapid test units $(25 \%, 187 / 749)$ than usual care $(39 \%, 374 / 951)$ units
(Table 3). Supplementary Table S5 provides the various indications for the administration of antibiotics to the newborns in both types of units. In three-quarters of such neonates (72\% rapid test, $73 \%$ usual care) commenced on antibiotics for suspected sepsis, the infection was subsequently ruled out and the antibiotics were discontinued (Supplementary Table S6). There were 3 neonatal deaths amongst 749 births in the rapid test units and 8 death in 951 births in the usual care units.

\section{Accuracy of rapid test for diagnosing maternal GBS colonisation}

Overall, 557 of the 722 (77\%) women in the rapid test units contributed results to the rapid intrapartum test (whether first or subsequent tests), 619 (86\%)

Table 3 Effects of a rapid test for GBS on neonatal exposure to antibiotics compared to usual care

\begin{tabular}{|c|c|c|c|c|}
\hline Outcome & $\begin{array}{l}\text { Rapid test, } n \\
\text { (\%) }\end{array}$ & $\begin{array}{l}\text { Usual care, } n \\
\text { (\%) }\end{array}$ & $\begin{array}{l}\text { Adjusted risk difference (95\% } \\
\mathrm{Cl}^{1}{ }^{1}\end{array}$ & $\begin{array}{l}\text { Adjusted relative risk (95\% } \\
\mathrm{Cl}^{2}\end{array}$ \\
\hline Neonatal (live births only) & $(n=748)$ & $(n=947)$ & & \\
\hline \multicolumn{5}{|l|}{ Antibiotics for any indication } \\
\hline Yes & $244(33 \%)$ & $412(44 \%)$ & $-0.13(-0.23,-0.02)$ & $0.71(0.54,0.95)$ \\
\hline No & $493(67 \%)$ & $534(56 \%)$ & & \\
\hline Missing & 11 & 1 & & \\
\hline \multicolumn{5}{|l|}{$\begin{array}{l}\text { Antibiotics for suspected early-onset } \\
\text { sepsis }\end{array}$} \\
\hline Yes & $187(25 \%)$ & $374(39 \%)$ & $-0.15(-0.26,-0.04)$ & $0.63(0.43,0.92)$ \\
\hline No & $548(75 \%)$ & $572(61 \%)$ & & \\
\hline Missing & 13 & 1 & & \\
\hline Neonatal mortality (all neonates)* & $(n=749)$ & $(n=951)$ & & \\
\hline Yes & $3(0.4 \%)$ & $8(0.84 \%)$ & & $0.48(0.10,2.21)$ \\
\hline No & $746(99.6 \%)$ & $943(99.16 \%)$ & & \\
\hline Missing & 0 & 0 & & \\
\hline
\end{tabular}

*Relative risk estimated from an unadjusted Poisson mixed effects model with robust standard errors, no model for the risk difference converged ${ }^{1}$ Risk difference; estimates $<0$ favour rapid test

${ }^{2}$ Relative risk; estimates $<1$ favour rapid test. Analyses adjusted for cluster size, unit birth rate, and estimated pre-trial IAP rates 
contributed to selective enrichment culture and 534 (74\%) contributed information on both tests (Fig. 2). The sensitivity of the rapid test was $86 \%$ (95\% CI $81-$ $91 \%)$, and the specificity was $89 \%$ (95\% CI $85-92 \%)$. The sensitivity of the rapid test was not statistically different from the target value of $90 \%(p=0.052)$ (Table 4$)$.

Sensitivity analyses that excluded women who were tested using only vaginal swabs and by excluding women who had vaginal cleansing or lubrication with an antimicrobial solution prior to the test yielded similar sensitivity and specificity estimates (Supplementary Table S7).

\section{Maternal and neonatal GBS colonisation}

The overall prevalence of maternal GBS colonisation was $43 \%$ ( $95 \%$ CI 39 to $48 \%$ ) by rapid test method and $41 \%$ (95\% CI $37-45 \%$ ) by selected enrichment culture (Supplementary Table S8). The overall rates of maternal GBS colonisation were similar when women who contributed a vaginal but not a rectal swab were excluded (Supplementary Table S8). The overall neonatal colonisation rate was $11 \%(49 / 445 ; 95 \%$ CI 8 to $14 \%)$ in the 445 babies assessed using selective enrichment culture of neonatal ear swabs. Neonatal colonisation was detected in one in five neonates born to women colonised with GBS as per the selective enrichment culture (19\%, $35 / 186)$ or rapid test $(21 \%, 38 / 184)$ (Table 5).

\section{Microbiological and antibiotic resistance profiles}

Overall, 117 women provided samples using vaginalrectal swabs to determine the antibiotic resistance profile of GBS and other bacteria, with 64 paired samples (63 mothers and 64 infants including one set of twins); 60 paired samples were available for molecular testing (59 mothers, 60 infants).

We isolated GBS in 33\% (39/117) maternal vaginalrectal and in two infant faecal (2/64) samples; 82\% (32/ 39) of maternal GBS isolates were tetracycline-resistant, 23\% (9/39) erythromycin-resistant and 18\% (7/39) were clindamycin-resistant. No penicillin-resistant maternal GBS isolates were identified. E. coli was isolated in $73 \%$ of the maternal samples (85/117). Half of all $E$. coli isolated were resistant to ampicillin (54\%, 46/85), 44\% (37/ $85)$ to amoxycillin/clavulanate, $25 \%(21 / 85)$ to trimethoprim/sulphamethozaxole, $6 \%(5 / 85)$ to ciprofloxacin, $5 \%$ $(4 / 85)$ to gentamicin and $4 \%(3 / 85)$ to extendedspectrum $\beta$-lactamase (ES $\beta \mathrm{L}) ; 21 \%$ (18/85) were resistant to three or more antibiotic classes (multi-resistant MR). Vancomycin-resistant enterococci (VRE) were isolated from one infant and one unrelated maternal sample. There was no methicillin-resistant Staphylococcus aureus (MRSA) isolated from mother or infant samples. Gramnegative antibiotic resistance genes were identified in the 128 samples from mother-infant pairs: TEM, CTX-M, SHV, OXA-23,48,51(like), CMY, IMI, VIM, MCR-1, DHA, GES, KPC and NDM. Only TEM and CTX-M were present in 10 or more maternal samples; SHV (which is carried by Klebsiella species predominantly) was found in 14 of 60 infant samples compared with only 5 of 59 maternal samples, possibly reflecting differences in mother and infant Klebsiella colonisation $(p<$ 0.05).

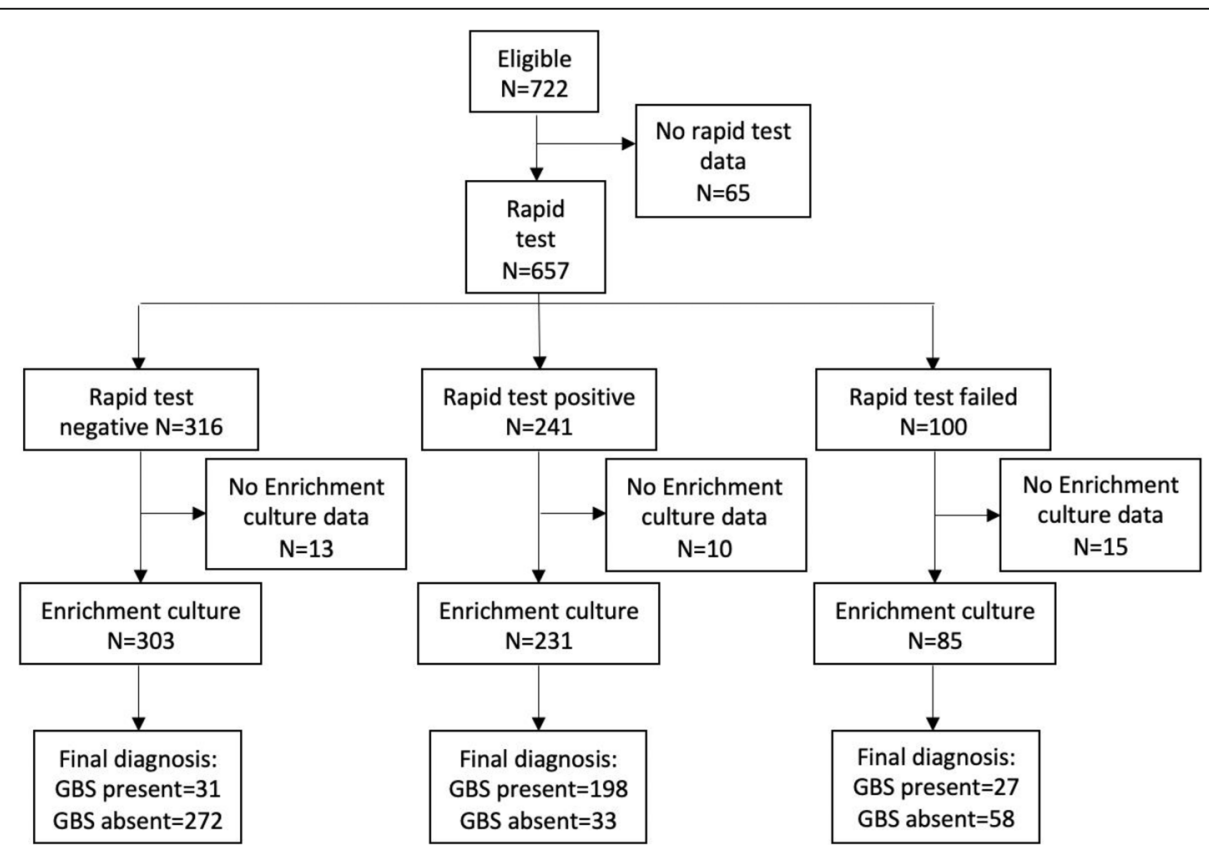

Fig. 2 Flow chart of the test data from rapid test and selective enrichment culture 
Table 4 Accuracy of rapid test in diagnosing maternal GBS colonisation status

\begin{tabular}{|c|c|c|c|c|c|}
\hline & & \multicolumn{2}{|c|}{ Selective enrichment culture } & \multirow{2}{*}{$\begin{array}{l}\text { Sensitivity } \\
(95 \% \mathrm{Cl})\end{array}$} & \multirow{2}{*}{$\begin{array}{l}\text { Specificity } \\
(95 \% \mathrm{Cl})\end{array}$} \\
\hline & & Positive & Negative & & \\
\hline \multirow[t]{2}{*}{ Rapid test } & Positive & $198(86 \%)$ & $33(11 \%)$ & $86 \%(81-91 \%)$ & $89 \%(85-92 \%)$ \\
\hline & Negative & 31 (14\%) & 272 (89\%) & & \\
\hline
\end{tabular}

There was a significant association for colonisation status in faecal samples of infants at 6-12 weeks of age and maternal vaginal-rectal colonisation status intrapartum for co-trimoxazole resistant and multi-resistant $E$. coli in both unadjusted and adjusted estimates (Table 6). The limited sample size precluded the convergence of a "full" model adjusted for all variables, so no estimates of relative risks or confidence intervals taking account of all variables are presented.

\section{Discussion}

In pregnant women with risk factors for early-onset GBS infection in their babies, the use of a point-of-care rapid test in labour to diagnose maternal GBS colonisation increased the administration of intrapartum antibiotics to prevent neonatal GBS infection by a small amount, compared with the usual care strategy of risk-factor based antibiotics administration, but with considerable uncertainty. The overall maternal exposure to antibiotics for any reason was not reduced with the use of the rapid test compared with usual care, whilst more women received an adequate duration of intrapartum antepartum prophylaxis in the rapid test units.

The absence of a reduction in intrapartum antibiotic prophylaxis through the implementation of a rapid testing strategy can be attributed to the following reasons. Firstly, although all women in usual care units should have been offered antibiotics if they had risk factors for neonatal early-onset GBS infection, this was only administered to $36 \%$ of women, which was lower than our expected estimate. This highlights the low adherence to the national guidelines, a situation that has changed little since a surveillance study in 2014-2015 where 44\% of women with risk factors received IAP [20]. Secondly, despite the negative rapid test results for GBS colonisation, clinicians still administered antibiotics for neonatal infection prophylaxis to $17 \%$ of women. We did not explore the reasons why clinicians were motivated to offer antibiotics in this circumstance.

Maternal GBS colonisation status is considered to be a predictor for neonatal sepsis and has been incorporated in prediction models for neonatal sepsis [21, 22]. A strategy of rapid testing for maternal GBS colonisation in clinical practice appeared to reduce the neonatal exposure to antibiotics, with fewer newborns diagnosed with suspected early-onset sepsis requiring antibiotics. Our findings indicate that neonatologists take into consideration the results of the rapid test to make decisions on whether to start antibiotic treatment in the newborn, thereby reducing unnecessary exposure of the newborns to antibiotics.

The rapid intrapartum test showed good sensitivity and specificity in diagnosing maternal GBS colonisation. Our test accuracy estimates reflect the expected accuracy of the test when implemented in routine clinical practice by midwives, unlike previous studies where the tests were invariably handled by laboratory staff.

There is some evidence to suggest an association between multi-drug resistant gram-negative bacterial colonisation in the mother with similar colonisation in the newborn at 6 weeks of age.

\section{Strengths and limitations}

Our randomised trial successfully engaged with multiple maternity units across different regions of the UK, trained healthcare professionals and implemented the rapid test in routine clinical practice. The maternal risk factors for neonatal early-onset GBS infection aligned with the national recommendations [10], and similar estimated rates of IAP in both strategy groups prior to the trial suggest consistent implementation of these recommendations. We recruited more than the required numbers of clusters, so that the study achieved a sufficient sample size, even when units dropped out prior to

Table 5 Rates of neonatal GBS colonisation in babies born to women tested for GBS using rapid test or selective enrichment culture

\begin{tabular}{lll}
\hline Maternal GBS colonisation status & & Neonatal colonisation present \\
\hline Selective enrichment culture* & Positive & $35 / 186(19 \%)$ \\
& Negative & $12 / 240(5 \%)$ \\
Rapid test** $^{*}$ & Positive & $38 / 184(21 \%)$ \\
& Negative & $7 / 203(3 \%)$ \\
\hline
\end{tabular}

${ }^{*}$ A total of 619 women had results for selective enrichment culture; 418 of these mothers had 426 neonates providing ear swabs

${ }^{* *}$ A total of 557 women had results for rapid test; 380 of these mothers had 387 neonates providing ear swabs 
Table 6 Relative risk of baby colonisation born to mothers colonised with antibiotic-resistant Escherichia coli and other resistance genes compared with non-colonised mothers

\begin{tabular}{|c|c|c|c|c|c|c|}
\hline \multirow[t]{2}{*}{ Outcome } & \multicolumn{6}{|c|}{ Relative risk of colonisation in babies at 6-12 weeks of age by maternal colonisation status ( $n=64$ babies) } \\
\hline & $\begin{array}{l}\text { Unadjusted } \\
\text { estimate }\end{array}$ & $\begin{array}{l}\text { Adjusted for } \\
\text { mode of } \\
\text { delivery }\end{array}$ & $\begin{array}{l}\text { Adjusted for } \\
\text { gestational age at } \\
\text { birth }\end{array}$ & $\begin{array}{l}\text { Adjusted for } \\
\text { neonatal antibiotic } \\
\text { use }\end{array}$ & $\begin{array}{l}\text { Adjusted for } \\
\text { maternal antibiotic } \\
\text { use }\end{array}$ & $\begin{array}{l}\text { Adjusted for } \\
\text { neonatal ICU } \\
\text { admission }\end{array}$ \\
\hline E. coli detected & $\begin{array}{l}1.3(0.70 \\
2.47)\end{array}$ & $1.29(0.68,2.46)$ & $1.35(0.72,2.52)$ & $1.31(0.70,2.46)$ & $1.32(0.70,2.49)$ & - \\
\hline $\begin{array}{l}\text { Ampicillin- } \\
\text { resistant E. coli }\end{array}$ & $\begin{array}{l}1.98(0.94 \\
4.16)\end{array}$ & $2.29(1.13,4.64)$ & $2.13(1.04,4.39)$ & $1.91(0.92,3.97)$ & $2.00(0.95,4.23)$ & - \\
\hline $\begin{array}{l}\text { Co-amoxiclav- } \\
\text { resistant E. coli }\end{array}$ & $\begin{array}{l}1.93(0.81 \\
4.58)\end{array}$ & $2.14(0.88,5.19)$ & $2.24(0.97,5.19)$ & $1.95(0.84,4.53)$ & $1.84(0.77,4.40)$ & - \\
\hline $\begin{array}{l}\text { Co-trimoxazole- } \\
\text { resistant E. coli }\end{array}$ & $\begin{array}{l}4.82(1.12, \\
20.8)\end{array}$ & $4.44(0.97,20.3)$ & $5.16(1.21,22.0)$ & $4.71(1.04,21.4)$ & $4.51(1.03,19.7)$ & $4.55(1.05,19.6)$ \\
\hline $\begin{array}{l}\text { Multiple } \\
\text { resistance in } E \text {. } \\
\text { coli }\end{array}$ & $\begin{array}{l}6.50(1.22 \\
34.7)\end{array}$ & $5.14(0.99,26.6)$ & $6.70(1.29,34.8)$ & $9.52(1.93,46.9)$ & $6.39(1.17,34.8)$ & $6.13(1.15,32.7)$ \\
\hline $\begin{array}{l}\text { TEM resistance } \\
\text { gene }\end{array}$ & $\begin{array}{l}1.71(0.96 \\
3.06)\end{array}$ & $1.83(1.01,3.33)$ & $1.92(1.07,3.46)$ & $1.89(1.07,3.34)$ & $1.67(0.91,3.06)$ & $1.87(1.01,3.46)$ \\
\hline $\begin{array}{l}\text { CTX-M resistance } \\
\text { gene }\end{array}$ & $\begin{array}{l}2.00(0.98 \\
4.06)\end{array}$ & $1.87(0.88,3.99)$ & $1.88(0.93,3.79)$ & $1.88(0.94,3.74)$ & $1.92(0.95,3.89)$ & $1.86(0.92,3.77)$ \\
\hline
\end{tabular}

Empty cells correspond to analyses that failed to converge and produce estimates. Estimates are obtained through log-binomial regression models in which the presence or absence of infant carriage is modelled by the presence or absence of maternal carriage alone and the value of the specified adjustment variable. The gestational age variable is continuous, and all other variables are binomial

recruitment. The cluster randomisation design avoided the risk of bias, since the availability of the rapid testing facility would make it difficult for healthcare professionals not to offer the test, given the rapid access to results. We provided rapid test machines only to units allocated to that strategy. We did not record whether women changed their intended place of birth due to knowledge of the randomised strategy.

In addition to the primary outcome of intrapartum antibiotics for prevention of early-onset neonatal GBS infection, we also reported other relevant outcomes such as maternal and neonatal exposure to antibiotics given for any reason, and rates of suspected earlyonset sepsis in newborns requiring antibiotics. We considered the sensitivity of our assumptions to a range of proportions in the usual care group and a range of ICC values which we believed to be quite conservative. The ICC calculated at the end of the trial at 0.06 (95\% CI $0.03-0.12)$ was within our assumption used in the sample size calculation. Our analysis of the primary outcome allowed for clustering at a maternity unit level as a random effect, corrected for the small number of clusters. The embedded test accuracy study allowed us to simultaneously assess the real-time accuracy of the test in clinical practice, in addition to minimising research waste. We minimised the risk of bias by performing the rapid tests and selective enriched culture tests independently and evaluated blindly to each other. The double-headed swab ensured that the index and reference tests were undertaken on contemporaneous samples.
The study had limitations. It is possible that eligible women may have been missed in the rapid test units, as unlike usual care units where the data were collected from recorded notes, the offer of a rapid test to the woman depended on the availability and training of staff. We observed potential differential ascertainment of eligible participants between the two strategies, with more women with recorded antenatal GBS colonisation recruited in the rapid test units, and with intrapartum fever in the usual care units. It is possible that women with known prior GBS colonisation may have been more likely to be offered the rapid test.

We were not able to blind the women and the clinical staff due to obvious differences in testing strategies. However, ascertainment of the primary outcome was objective based on recorded administration of antibiotic use. Since the indications for antibiotic administration were ascertained from the clinical records in the usual care units, it is possible that some of the antibiotics administered for GBS prophylaxis may have been classified under a different indication. This is similar to the finding of the Royal College of Obstetricians and Gynaecologists' audit that highlighted problems with interpreting the definition of intrapartum antibiotic prophylaxis and its indication [23].

Although we had fewer women with paired samples for the rapid test and selective enrichment culture than our projected sample size, the actual rate of maternal GBS colonisation was higher than anticipated. In a previous comparable study, $89 \%$ of rapid tests yielded a result, whereas we observed a lower yield of $77 \%$ in our study 
[24]. There is potential for spectrum bias, as the rapid test was only done in women with risk factors for earlyonset GBS infection in the baby, although previous work had shown similar post-test probabilities for maternal colonisation in women with and without risk factors [12]. We did not collect information on maternal ethnicity. Our findings on the association between infant antibiotic resistance profile and maternal colonisation status were limited by the small numbers of paired samples.

\section{Comparison to other studies}

To date, there have been no randomised trials evaluating the use of rapid GBS test in routine clinical practice compared to usual care. A prospective cohort in France that compared the performance of intrapartum GeneXpert test to antenatal microbiological screening for maternal GBS colonisation, reported that universal rapid intrapartum test had the potential to have an absolute risk reduction of $0.925 \%$, equivalent to 108 more women needing to be tested in labour and provided with intrapartum prophylaxis to prevent a single case of earlyonset GBS neonatal infection that would be missed by the culture-based testing at 35-37 weeks [24]. Compared with the French study where $89 \%$ of rapid tests yielded a result, we observed a lower yield of $77 \%$ in our study.

A meta-analysis of 15 studies on the accuracy of various PCR platforms to detect maternal GBS colonisation reported produced pooled sensitivity of $93.7 \%$ and specificity of $97.6 \%$ [25]. These estimates were higher than what was observed in the GBS2 study, although sensitivities within primary studies using the GeneXpert system ranged from 83.0 to $98.5 \%$. Our findings indicate the sensitivity of the GeneXpert test is consistent with late third trimester selective enrichment culture in determining the colonisation status at birth [7]. In our study, the rates of GBS colonised babies born to mothers colonised with GBS was lower (20\%) than the $36 \%$ reported in a previous meta-analysis of six small studies (308 women and 117 babies colonised) [1], which may be influenced by the treatment provided in our study. Although we did not assess the acceptability of the rapid test to women in this study, in our previous UK GBS rapid testing study, we found that $94 \%(984 / 1043)$ of mothers were happy or very happy with the way the swabs were taken, and $94 \%$ $(975 / 1036)$ confident in its use in routine care [12].

\section{Conclusion}

The use of a point-of-care rapid test in clinical practice to detect maternal GBS colonisation does not reduce the rate of antibiotics administered to the mother with risk factors to prevent neonatal early-onset GBS infection compared to the usual care policy of offering antibiotics based on only risk factors. The newborns appear to be less exposed to antibiotics with the rapid test than usual care. The GeneXpert rapid test has acceptable accuracy in detecting maternal GBS colonisation. Our sub-study highlights the potential for increased risk of multi-drug resistant $E$. coli in infants at 6-12 weeks of age in mothers colonised with such multi-drug resistant bacteria. The ongoing GBS3 (ISRCTN49639731) trial, which is comparing routine intrapartum rapid testing and antenatal microbiological testing in all women intending to deliver vaginally on the incidence of neonatal sepsis, will provide crucial information on the benefits of universal testing.

\section{Abbreviations}

$\mathrm{Cl}$ : Confidence interval; ES $\beta$ L: Extended-spectrum $\beta$-lactamase producing Enterobacterales; GBS: Group B Streptococcus; IAP: Intrapartum antibiotic prophylaxis; ICC: Intra-cluster coefficient; MRSA: Staphylococcus aureus; VRE: Vancomycin-resistant enterococci

\section{Supplementary Information}

The online version contains supplementary material available at https://doi. org/10.1186/s12916-021-02202-2.

Additional file 1 : Table S1. Antibiotic administration by GBS rapid test result. Table S2. Provision of antibiotic for different clinical reasons, by GBS test result. *Women can have more than one reason given for receiving intrapartum antibiotics. Table S3. Maternal antibiotic use in rapid test and usual care maternity units. ${ }^{*}$ Unadjusted analysis due to lack of convergence with the adjusted model. ${ }^{*}$ Estimated through an adjusted Poisson model with robust standard errors, estimates should be interpreted with caution as the covariance matrix for the random effects is not positive definite. Table S4. Use of GBS intrapartum antibiotic prophylaxis by risk factor subgroups. Table S5. Comparison of the reasons for neonatal antibiotic administration. * For 85 babies, there were $>1$ reason for receiving antibiotics; $\wedge$ For 129 babies, there were $>1$ reasons for receiving antibiotics; $\mathrm{N}$ is number of babies who received antibiotics and indication provided. Table S6. Management of babies administered IV antibiotics for suspected early neonatal sepsis. Table S7. Sensitivity analysis of accuracy of rapid test to diagnose GBS colonisation. Table S8. Prevalence of GBS maternal colonisation by test.

\section{Acknowledgements}

Collaborative group:

Khaled Ahmed, Julie Dodds and Maria D'Amico, who coordinated the study; Kostas Tryposkiadis, who was responsible for the randomisation algorithm; Angela Whiley, who coordinated the laboratory analyses; and Patrick Moore and Ruvimbo Lorraine Munetsi, who performed a parallel economic analysis. Site principal investigators: Pallavi Karkhanis, Anne Deans, Sanjula Sharma, Gemma Wright, Manjula Subramanian, Irene Ray, Dibyenda Datta, Lauren Lacey, Johnathon Pepper, Ruth Mason, Neil Shah, Katharina Anwar, Neena Navaneetham, Shad Husain, Phillip Bennett, Geraldine Masson, Hristina Raykova, Matthew Hogg, Bashir Dawalatly, Lakshmi Thirumalaikumar and Kate Townsend, research midwives and maternity unit staff. We would like to thank Gerry Collins for facilitating the study conduct.

GBS2 Steering and Data Monitoring Committee members: Paul Heath, Kerry Hood, Stavros Petrou, Ben Stenson, Sarah McMullen, Julia Saunders, Alison Stanley, Stephen Walters, Patrick Bossuyt, Ruth Gilbert and Rhona Hughes. Parent and public involvement:

The Group B Strep Support (GBSS https://gbss.org.uk) charity provided input at all stages of the study including identification of research question, development of study protocol, study conduct and interpretation of the results. Their input was crucial to ensure the acceptability of the study to women and healthcare professionals. They were involved in promoting the engagement of maternity units to the study and prompted us to provide study-specific testing information leaflets to all eligible women. All patientfacing materials and approach for the consent pathway were discussed and reviewed by Katie's Team, an East London-based group which includes 
members of the public who are parents and interested in research. Direct feedback to participants is not possible as no identifiable details were collected.

\section{Authors' contributions}

JPD, MW, MM, SH, JG, TER, JP, JJD, KH, KSK and ST conceived the overall project and were responsible for the funding acquisition. JPD, JB, MW, MM JG, TER, JJD, KH, KSK and ST contributed to the study methodology. JB and AG undertook the statistical analysis. JP led on the parent and public involvement. JPD, ED, KH and ST drafted the report. All authors reviewed and approved the final manuscript. JPD and ST accept full responsibility for the work and/or the conduct of the study, had access to the data and controlled the decision to publish.

\section{Funding}

GBS2 was funded by the National Institute for Health Research (NIHR) Health Technology Assessment programme (13/82/04). The funder had no role in the design and conduct of the study; the collection, management, analysis and interpretation of the data; the writing of the manuscript; or the decision to submit the manuscript for publication. The views expressed are those of the authors and not necessarily those of the NHS, the NIHR or the Department of Health and Social Care.

JJD is supported by the NIHR Birmingham Biomedical Research Centre at the University Hospitals Birmingham NHS Foundation Trust and the University of Birmingham. KSK is a Distinguished Investigator funded by the Beatriz Galindo (senor modality) Program grant given to the University of Granada by the Ministry of Science, Innovation, and Universities of the Spanish Government.

\section{Availability of data and materials}

We shall make data available to the scientific community with as few restrictions as feasible, whilst retaining exclusive use until the publication of major outputs. All data requests should be submitted to the corresponding author for consideration.

\section{Declarations}

\section{Ethical approval and consent to participate}

This study was approved by the West Midlands - Edgbaston Multi-centre Research Ethics Committee (16/WM/0036), including waiver of individual research consent. GBS2 was sponsored under the Research Governance Framework for Health and Social Care (2015) by Queen Mary, University of London.

\section{Consent for publication}

No identifiable data is presented.

\section{Competing interests}

All authors have completed the ICMJE uniform disclosure form at wwW. icmje.org/coi_disclosure.pdf. JP is the Chief Executive of Group B Strep Support, a Charity working to stop GBS in babies. JP received support from Cepheid to attend an academic conference in 2016, from Pfizer to attend a summit for vaccine advocacy stakeholders in the USA in 2019 and from iCONSENT to attend workshops in London in 2018 and Brussels in 2019 regarding consent for vaccine trials. JP was a member of the Department of Health research prioritisation expert group in 2016. JP and JG are members of the NICE guideline update committee for Neonatal infection: antibiotics for prevention and treatment (update). JG was a member of the NICE Diagnostics Advisory Committee that produced DG38 Rapid tests for group A streptococcal infections in people with a sore throat (which included assessment of the Cepheid GeneXpert Xpert ${ }^{\oplus}$ Xpress Strep A test) (Published November 2019). JPD, JP and JG are grant holders for HTA 17/86/06 (GBS3), a cluster randomised trial of routine screening for GBS. JPD and JP are grant holders for a study to determine a serocorrelate of immune protection against GBS.

The other authors declare that they have no competing interests.

\section{Author details}

${ }^{1}$ Nottingham Clinical Trials Unit, School of Medicine, University of Nottingham, Nottingham NG7 2RD, UK. ${ }^{2}$ Birmingham Clinical Trials Unit, College of Medical and Dental Sciences, University of Birmingham,
Birmingham, UK. ${ }^{3}$ Barts Health NHS Trust, London, UK. ${ }^{4}$ Queen Mary University of London, London, UK. ${ }^{5}$ Birmingham Women's \& Children's NHS Foundation Trust, Birmingham, UK. ${ }^{6}$ Institute of Applied Health Research, College of Medical and Dental Sciences, University of Birmingham, Birmingham, UK. ${ }^{7}$ Group B Strep Support, JYW House, Bridge Road, Haywards Heath, UK. ${ }^{8} \mathrm{NIHR}$ Birmingham Biomedical Research Centre, University Hospitals Birmingham NHS Foundation Trust and University of Birmingham, Birmingham, UK. 9Department of Preventive Medicine and Public Health, University of Granada, Granada, Spain. ${ }^{10}$ Institute of Metabolism and System Research, College of Medical \& Dental Sciences, University of Birmingham, Birmingham, UK.

Received: 21 June 2021 Accepted: 29 November 2021 Published online: 14 January 2022

\section{References}

1. Colbourn T, Gilbert R. An overview of the natural history of early onset group B streptococcal disease in the UK. Early Hum Dev. 2007;83(3):149-56. https://doi.org/10.1016/j.earlhumdev.2007.01.004.

2. O'Sullivan CP, Lamagni T, Patel D, Efstratiou A, Cunney R, Meehan M, et al. Group B streptococcal disease in UK and Irish infants younger than 90 days, 2014-15: a prospective surveillance study. Lancet Infect Dis. 2019;19(1):8390. https://doi.org/10.1016/S1473-3099(18)30555-3.

3. Madrid L, Seale AC, Kohli-Lynch M, Edmond KM, Lawn JE, Heath PT, et al. Infant group B Streptococcal disease incidence and serotypes worldwide: systematic review and meta-analyses. Clin infect dis. 2017;65(suppl_2):S160-s72.

4. Fairlie T, Zell ER, Schrag S. Effectiveness of intrapartum antibiotic prophylaxis for prevention of early-onset group B streptococcal disease. Obstet Gynecol. 2013;121(3):570-7. https://doi.org/10.1097/AOG.0b013e318280d4f6.

5. Ohlsson A, Shah VS, Stade BC. Vaginal chlorhexidine during labour to prevent early-onset neonatal group B streptococcal infection. Cochrane Database Syst Rev. 2014;12:CD003520. https://doi.org/10.1002/14651858. CD003520.pub3.

6. Prevention of group B Streptococcal early-onset disease in newborns. ACOG Committee Opinion Summary, Number 797. Obstet Gynecol. 2020;135(2): 489-92. https://doi.org/10.1097/AOG.0000000000003669.

7. Valkenburg-van den Berg AW, Houtman-Roelofsen RL, Oostvogel PM, Dekker FW, Dörr PJ, Sprij AJ. Timing of group B streptococcus screening in pregnancy: a systematic review. Gynecol Obstet Invest. 2010;69(3):174-83. https://doi.org/10.1159/000265942.

8. Di Renzo GC, Melin P, Berardi A, Blennow M, Carbonell-Estrany X, Donzelli GP, et al. Intrapartum GBS screening and antibiotic prophylaxis: a European consensus conference. J Matern Fetal Neonatal Med. 2015;28(7):766-82. https://doi.org/10.3109/14767058.2014.934804.

9. National Screening Committee. The UK NSC recommendation on Group B Streptococcus screening in pregnancy. UK: Public Health England; 2017.

10. Prevention of early-onset neonatal group B Streptococcal disease. London: Royal College of Obstetricians and Gynaecologists; 2017.

11. National Institute of Health and Clinical Excellence. Antibiotics for the prevention and treatment of early-onset neonatal infection. London: National Institute of Health and Clinical Excellence; 2012.

12. Daniels J, Gray J, Pattison H, et al. Rapid testing for group B streptococcus during labour: a test accuracy study with evaluation of acceptability and cost-effectiveness. Health Technol Assess. 2009;13(42):1-154.

13. Hemming K, Kasza J, Hooper R, Forbes A, Taljaard M. A tutorial on sample size calculation for multiple-period cluster randomized parallel, cross-over and stepped-wedge trials using the Shiny CRT Calculator. International journal of epidemiology. 2020;49(3):979-95. https://doi.org/10.1093/ije/dyz23 7.

14. Kenward MG, Roger JH. Small sample inference for fixed effects from restricted maximum likelihood. Biometrics. 1997;53(3):983-97. https://doi. org/10.2307/2533558.

15. Li P, Redden DT. Comparing denominator degrees of freedom approximations for the generalized linear mixed model in analyzing binary outcome in small sample cluster-randomized trials. BMC Med Res Methodol. 2015;15(1):38. https://doi.org/10.1186/s12874-015-0026-x.

16. UK Standards for Microbiology Investigations - Detection of carriage of group B Streptococci (Streptococcus agalactiae). Public Health England; 2015 (updated Jun 2018).

17. Colbourn T, Asseburg C, Bojke L, Philips Z, Claxton K, Gilbert R. Prenatal screening and treatment strategies to prevent group B Streptococcal and 
other bacterial infections in early infancy: cost effectiveness and expected value of information analyses. Health Technol Assess. 2007;7(29):1-226.

18. Clopper CJ, Pearson ES. The use of confidence or fiducial limits illustrated in the case of the binomial. Biometrika. 1934;26(4):404-13. https://doi.org/10.1 093/biomet/26.4.404.

19. Matuschek E, Brown DF, Kahlmeter G. Development of the EUCAST disk diffusion antimicrobial susceptibility testing method and its implementation in routine microbiology laboratories. Clinical microbiology and infection. 2014;20(4):0255-66.

20. British Paediatric Surveillance Unit. Annual Report. 2015-2016.

21. Puopolo KM, Benitz WE, Zaoutis TE. Management of neonates born at $\geq 35$ 0/7 weeks' gestation with suspected or proven early-onset bacterial sepsis. Pediatrics. 2018;142(6):e20182894. https://doi.org/10.1542/peds.2018-2894.

22. Puopolo KM, Draper D, Wi S, Newman TB, Zupancic J, Lieberman E, et al. Estimating the probability of neonatal early-onset infection on the basis of maternal risk factors. Pediatrics. 2011;128(5):e1155-63. https://doi.org/10.1 542/peds.2010-3464.

23. Royal College of Obstetricians and Gynaecologists. Audit of current practice in preventing early-onset neonatal group B streptococcal disease in the UK. London; 2015.

24. El Helali N, Nguyen JC, Ly A, Giovangrandi Y, Trinquart L. Diagnostic accuracy of a rapid real-time polymerase chain reaction assay for universal intrapartum group B Streptococcus screening. Clin Infect Dis. 2009;49(3): 417-23. https://doi.org/10.1086/600303.

25. Feuerschuette OHM, Silveira SK, Cancelier ACL, da Silva RM, Trevisol DJ, Pereira JR. Diagnostic yield of real-time polymerase chain reaction in the diagnosis of intrapartum maternal rectovaginal colonization by group $B$ Streptococcus: a systematic review with meta-analysis. Diagn Microbiol Infect Dis. 2018;91 (2):99-104. https://doi.org/10.1016/j.diagmicrobio.2018.01. 013.

\section{Publisher's Note}

Springer Nature remains neutral with regard to jurisdictional claims in published maps and institutional affiliations.

Ready to submit your research? Choose BMC and benefit from:

- fast, convenient online submission

- thorough peer review by experienced researchers in your field

- rapid publication on acceptance

- support for research data, including large and complex data types

- gold Open Access which fosters wider collaboration and increased citations

- maximum visibility for your research: over $100 \mathrm{M}$ website views per year

At $\mathrm{BMC}$, research is always in progress.

Learn more biomedcentral.com/submissions 